\title{
X-ray properties of rotation-powered pulsars
}

\author{
George Pavlov ${ }^{1}$ and Oleg Kargaltsev ${ }^{2}$ \\ ${ }^{1}$ Pennsylvania State University, United States \\ email: pavlov@astro.psu.edu \\ ${ }^{2}$ University of Florida \\ email: oyk100@astro.ufl.edu
}

\begin{abstract}
Radiation of most of the known pulsars is powered by the loss of their rotational energy and angular momentum. Thanks to the Chandra and XMM-Newton observatories, X-ray emission has been detected from over 100 rotation-powered pulsars in the last 12 years. I will overview the current results of X-ray observations of rotation-powered pulsars, including the evolution of the X-ray properties, thermal and magnetospheric components of X-ray emission, and connection between the X-ray emission and emission at other wavelengths.
\end{abstract}

